\title{
Interactive comment on "Non-linear Effects in Electromagnetic Wave Activity Observed in the RELEC Experiment on-board Vernov Mission" by Mikhail I. Panasyuk et al.
}

Mikhail I. Panasyuk et al.

bogovit@rambler.ru

Received and published: 13 February 2019

Authors are much pleasured to referees for comprehensive and very useful remarks. According to these remarks we re-elaborated the paper quite significantly. All re-written parts of article are picked-out by yellow in the supplemented *.pdf file. Below we listed referee's remarks and our commentaries.

"The author must show a clear no relation between the abnormal wave phenomena and non-seismic activity. I suggest further validation on the wave phenomena in periods for both seismic and non-seismic activities. I think that Section 4 "discussion" is not well discussed and Section 5 "conclusion" is not meaningful (just repeat). I suggest that 
both sections must be improved with more detailed explanations, discussions, and validation analyses. In my opinion, it requires major revision."

ANGEOD

We added the Section 4 "Discussion" by more detailed analysis of wave phenomena in periods of seismic activity. In particular, it was shown by adding of corresponding references that a high-frequency tail at the whistler event reaching $25 \mathrm{kHz}$ indicates the presence of a low conductivity area at the lower ionosphere in the zone of lightning discharges, which contributes to the attenuation of the VLF wave absorption, and that the growth of high-frequency atmospheric penetration is realized in the region of earthquake before it. This was also illustrated by Fig. 11, which was also re-elaborated. References, which show the presence of thunderstorm activity for a few days or hours before the earthquakes, were also added. Thus, it was concluded that analysed whistler may be of lightning origin occurred on the eve of earthquake. Presented in Fig.12 a map of regional seismic activity after the discussed whistler was also significantly redone.

Specific comments

Abstract.

If "RELEC" is an abbreviation/acronym, please define accordingly.

Done - acronym "RELEC" was omitted from Abstract and defined in Section 1 "Introduction"

The figurative expression "swallowtail type" is not clear in abstract. Please modify using more suitable expression without no figure information.

Done - "swallowtail type" was replaced on "which looked like a high frequency tail rising" 4. Discussion.

As pointed out above, the authors must add the validity analysis for the abnormal wave phenomena related to the seismic activity using a long period including before and

Printer-friendly version

Discussion paper
Interactive

comment 
after the focusing seismic activity. If the authors do not show the validity analysis, the relation between the wave phenomena and seismic activity is not supported by any demonstration.

For validation of that abnormal wave phenomena may be connected with seismic activity just Fig. 11 is used. It presents partially dispersed whistler spectra in periods of seismic quiet time and of seismic activity. This figure nudicates that increasing of number of whistler events with frequencies higher than $\sim 5 \mathrm{kHz}$ is clearly higher in periods of seismic activity.

Fig. 10 Please describe Fig. 10 in more details. What are the red line, gray and aqua circles?

Fig. 10 was renumbered in Fig. 12. It was re-elaborated significantly. The new map with Earthquakes near Kamchatka region is presented. All lines and marks was described in the text and Figure capture.

Fig. 11 Why is Fig. 11 important in this study? The results would be analyzed in a different satellite, different periods, different locations in contrast to the focusing event. If Fig. 11 is important in this study, please show the detailed information on the analysis (data period, observed location, data specifications etc.).

Fig. 11 is really important for validation of that abnormal wave phenomena may be connected with seismic activity (see above). We think that reference on Intercosmos 24 experiment, which is given in the text is enough for information about this experiment.

p.11 line 29 What is the nonlinear effect in this study? I could not find discussion on the specific nonlinear effects on the wave phenomena. If a nonlinear effect is important, the author must discuss the specific effects on the nonlinear effects on the wave phenomena.

The nonlinear effect in our study means nonlinear dependence of the frequency from time. The Section 4 "Discussion" is added by special spectral analysis based on so- 
called bi-spectra. Its results are also discused in the Section 5 "Conclusion".

Technical corrections and typos.

ANGEOD

The reviewer mentions that the references on this study are inadequate. Please add adequate references. (For examples) p. 1 line 36 Add a reference on TLEs.

The reference on TLE is omitted.

Interactive

comment

p.1 line 41 Add a reference on the effects of high energy particle on electric devices.

Effects of high energy particles are also omitted.

p.1 line 42 Add a reference on precipitation of high energy particles.

Discussion about precipitation of high energy particles is omitted.

p. 1 line 54 Add a reference on the wave-particle interactions.

Done

p.2 line 3 Add a reference on the whistler mode waves.

Done

p.2 line 29 Add a reference on sferics.

Done

p.2 line 34 Add a reference on frequency distribution of sferic spectra.

Done

Please add the axis label and the unit for all figures with a sufficient font size. The font size looks like small in the most of present figures.

Printer-friendly version

Done

Add the information on satellite location for Figs. 2, 3, 7a, 8, and 9ab as the same with 
Fig. 4.

We add the separate figure with satellite location for all times for which events were ANGEOD discussed in the paper.

p.9, line 13 superscript " $1 / 2$ "

Interactive

Done

comment

Please also note the supplement to this comment:

https://www.ann-geophys-discuss.net/angeo-2018-119/angeo-2018-119-AC1-

supplement.pdf

Interactive comment on Ann. Geophys. Discuss., https://doi.org/10.5194/angeo-2018-119, 2018. 